\title{
Recurrence-based information processing in gene regulatory networks
}

Cite as: Chaos 28, 106313 (2018); https://doi.org/10.1063/1.5039861

Submitted: 11 May 2018 . Accepted: 20 August 2018 . Published Online: 11 October 2018

Marçal Gabalda-Sagarra (D), Lucas B. Carey, and Jordi Garcia-Ojalvo (D)
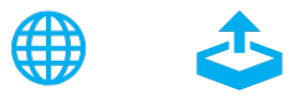

View Online

Export Citation

\section{ARTICLES YOU MAY BE INTERESTED IN}

Harnessing stochasticity: How do organisms make choices?

Chaos: An Interdisciplinary Journal of Nonlinear Science 28, 106309 (2018); https:// doi.org/10.1063/1.5039668

Introduction to Focus Issue: Nonlinear science of living systems: From cellular mechanisms to functions

Chaos: An Interdisciplinary Journal of Nonlinear Science 28, 106201 (2018); https:// doi.org/10.1063/1.5065367

Complexity and irreducibility of dynamics on networks of networks

Chaos: An Interdisciplinary Journal of Nonlinear Science 28, 106306 (2018); https:// doi.org/10.1063/1.5039483

\section{AIP Author Services English Language Editing}




\title{
Recurrence-based information processing in gene regulatory networks
}

\author{
Marçal Gabalda-Sagarra, Lucas B. Carey, and Jordi Garcia-Ojalvo a) \\ Department of Experimental and Health Sciences, Universitat Pompeu Fabra, Barcelona Biomedical Research \\ Park, 08003 Barcelona, Spain
}

(Received 11 May 2018; accepted 20 August 2018; published online 11 October 2018)

\begin{abstract}
Cellular information processing is generally attributed to the complex networks of genes and proteins that regulate cell behavior. It is still unclear, however, what are the main features of those networks that allow a cell to encode and interpret its ever changing environment. Here, we address this question by studying the computational capabilities of the transcriptional regulatory networks of five evolutionary distant organisms. We identify in all cases a cyclic recurrent structure, formed by a small core of genes, that is essential for dynamical encoding and information integration. The recent history of the cell is projected nonlinearly into this recurrent reservoir of nodes, where it is encoded by its transient dynamics, while the rest of the network forms a readout layer devoted to decode and interpret the high-dimensional dynamical state of the recurrent core. In that way, gene regulatory networks act as echo-state networks that perform optimally in standard memory-demanding tasks, with most of their memory residing in the recurrent reservoir. The biological significance of these results is analyzed in the particular case of the bacterium Escherichia coli. Our work thus suggests that recurrent nonlinear dynamics is a key element for the processing of complex time-dependent information by cells. Published by AIP Publishing. https://doi.org/10.1063/1.5039861
\end{abstract}

Cells must monitor the dynamics of their environment continuously, in order to adapt to present conditions and anticipate future changes. But anticipation requires processing temporal information, which in turn requires memory. Here, we propose that cells of five evolutionary distant organisms can perform such dynamical information processing via the reservoir computing paradigm. According to this concept, a structure with recurrent (cyclic) paths, known as the reservoir, stores in its nonlinear dynamics a record of the cell's recent history. A much simpler feedforward structure then reads and decodes that information. We show in particular that the architecture of the transcriptional gene regulatory network of the bacterium Escherichia coli allows it to store complex time-dependent signals in a biologically realistic manner.

\section{INTRODUCTION}

The survival of any cell, either as an individual entity or as part of a multicellular organism, depends on its capacity to respond to changes in the environment. In a wide variety of situations such as stress responses, morphogen-driven embryogenesis, immune reactions, and metabolic adaptations to varying energy sources, cells need to sense multiple signals in their surroundings, integrate them, and activate an adequate response. Orchestrating the best possible response with the right intensity is crucial, but so is doing it at the right moment and promptly enough. The importance of timing and speed implies that cells able to anticipate changes in the environment have a critical advantage.

Although many changes in the environment are stochastic from the point of view of a cell, many others are predictable.

a)Electronic mail: jordi.g.ojalvo@upf.edu
In many cases, the likelihood of future events is encoded by the recent history of the cell's environment. In these cases, the ability to use the temporal character of the information is clearly beneficial. Periodic changes in the environment, for example, can be anticipated through molecular oscillators or cellular clocks, as seen in the way most organisms on earth anticipate daily light-dark cycles, ${ }^{1}$ including relatively simple cyanobacteria. ${ }^{2,3}$ Another example is given by groups of events that tend to occur together or in a specific order. This kind of association, for instance, allows the bacterium Escherichia coli to prepare for oxygen depletion when it senses an increase in temperature, an indication that it has been ingested by a mammal. ${ }^{4}$ Similarly, enterobacteria anticipate sequential changes in sugars as they pass through the intestinal tract, and yeast cells expect a specific sequence of stresses during alcoholic fermentation. ${ }^{5}$ Pathogenic microbes are also known to detect variations in their environment to anticipate changes in the host-pathogen interaction cycle., Furthermore, experimental evolution studies have shown that predictive environmental sensing can evolve in relatively short periods of time in a laboratory setting. ${ }^{8}$

Beyond the ability to associate concurrent events, recent studies have shown that microbes exhibit both short- and longterm memory. The stress response of Bacillus subtilis, for example, depends not only on the condition in which it is currently growing but also on past growth conditions. ${ }^{9}$ However, the way in which this record of previous history-i.e., memory-is integrated and stored in cells is not yet fully understood. Knowledge of the conceptual limits of this cellular memory is also scarce. Since memory is a key limitation to recognizing temporal structures, the prediction capabilities of cells remain to be delimited as well.

While the passive prediction mechanisms of cells usually involve small circuits with only a handful of biomolecules (such as in genetic clocks), cellular adaptability relies in 
general on a complex network of interactions between genes and proteins, frequently at the level of transcriptional regulation. ${ }^{10,11}$ Here, we hypothesize that the global structure of this network determines how memory is encoded. Specifically, we aim to establish how gene regulatory networks integrate complex inputs, and especially how they process time-varying information. By analyzing different organisms, we propose that gene regulatory networks encode temporal information in a state-dependent manner: the recent history of the cell is encoded in the complex transient dynamics of the network, through the interaction between its internal state (which depends on its recent past) and the external inputs that are currently being received by the system. In the field of neural networks, this strategy has come to be known as reservoir computing $^{12}$ (encompassing the concepts of echo-state network from machine learning ${ }^{13}$ and liquid-state machine from computational neuroscience). ${ }^{14}$

Reservoir computing is a paradigm that allows processing of temporal information while featuring a very efficient learning process. Its key characteristic is that it separates memory encoding and prediction in two different network substructures (Fig. 1). The first substructure, known as the reservoir, contains recurrent connections (i.e., cyclic paths) and encodes information by projecting the stimulus nonlinearly into a high-dimensional space. ${ }^{15}$ The recurrent network of the reservoir allows it to retain information for a certain time, providing fading memory to the system. The second substructure, the readout layer, is a feedforward structure (i.e., a directed acyclic graph) placed downstream of the reservoir. This readout layer uses the history record encoded in the state of the reservoir to make a prediction or classification. Feedforward structures, lacking cyclic paths, are much easier to train

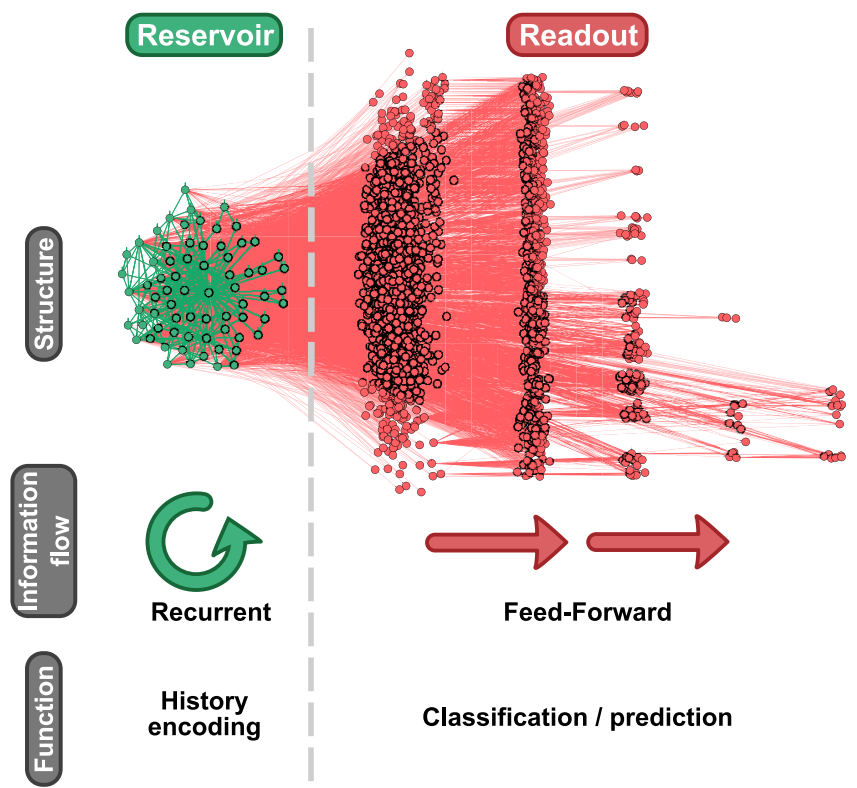

FIG. 1. Structural and functional organization of reservoir computing. The reservoir (green) is a subgraph with cyclic paths that can maintain a record of the recent history in its dynamics. The readout (red) is a directed acyclic subgraph that reads the information encoded in the reservoir state to perform a given task. The structures shown correspond to the Escherichia coli network (see Results). The nodes in the readout are grouped by the length of the longest path reaching them from the reservoir. (i.e., to adapt the strength of the interactions to produce the expected dynamics). This separation of roles allows the training process to be focused solely on the readout, giving this system the computational power of a recurrent network combined with the ease of training of a feedforward architecture. ${ }^{15}$ Furthermore, by adding independent readouts the system avoids catastrophic interference, i.e., it can incorporate additional tasks without interfering with the existing ones. ${ }^{16}$

Here, we propose that transcriptional networks can operate according to the reservoir computing paradigm. To test this conjecture, we first analyze the topology of the gene regulatory networks of five evolutionary distant organisms and examine how efficient these networks are at encoding and processing time-varying signals. Next, we show that the memory capacity of the networks is mainly located in their recurrent reservoirs. We then study the biological significance of these results in the particular case of Escherichia coli. To that end, we first consider biologically realistic inputs, in the form of different types of stress signals affecting this organism, and investigate how the information arriving through the corresponding pathways can be stored in the reservoirs. We also show that the readout layer can be trained in a biologically realistic manner through evolutionary processes. Finally, we propose several experimental approaches to validate the hypothesis put forward in this paper. The code and data presented in this work are freely available at https://github.com/mgab/GRN-Reservoirs.

\section{STRUCTURAL NETWORK ANALYSIS}

We analyzed the transcriptional networks of five evolutionary distant organisms: Bacillus subtilis (data obtained from DBTBS), ${ }^{17}$ Escherichia coli (EcoCyc), ${ }^{18}$ Saccharomyces cerevisiae (YEASTRACT), ${ }^{19}$ Drosophila melanogaster (modENCODE), ${ }^{20}$ and Homo sapiens (ENCODE). ${ }^{21}$ The degree distributions of all the networks exhibit a non-trivial structure, resembling in most of the cases
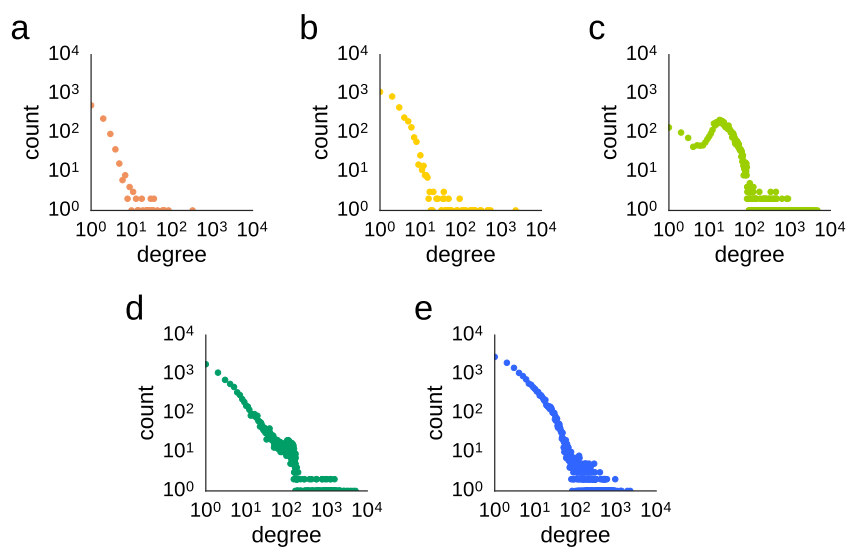

FIG. 2. Degree distribution of the gene regulatory networks: Bacillus $s u b$ tilis (a), Escherichia coli (b), Saccharomyces cerevisiae (c), Drosophila melanogaster (d), and Homo sapiens (e). In the case of Saccharomyces cerevisiae, the deviation observed in the degree distribution plot (panel c) is thought to be an artefact: since most of the data in this database come from compiling a large number of low throughput studies, nodes with lower degree can be expected to be under-represented, as studies tend to focus on genes involved in more regulatory interactions. 
TABLE I. General properties of the gene regulatory networks and their recurrent cores.

\begin{tabular}{lcccc}
\hline \hline & Nodes & Edges & $\begin{array}{c}\text { Self } \\
\text { loops }\end{array}$ & $\begin{array}{c}\text { Mean } \\
\text { degree }\end{array}$ \\
\hline Whole graph & & & & \\
$\begin{array}{l}\text { B. subtilis } \\
\text { E. coli }\end{array}$ & 886 & 1358 & 49 & 3.06 \\
S. cerevisiae & 3236 & 8366 & 126 & 5.17 \\
$\begin{array}{l}\text { D. melanogaster } \\
\text { H. sapiens }\end{array}$ & 6725 & 201972 & 197 & 60.06 \\
Recurrent core & 9432 & 231174 & 0 & 49.01 \\
$\begin{array}{l}\text { B. subtilis } \\
\text { E. coli }\end{array}$ & 16354 & 163271 & 28 & 19.96 \\
S. cerevisiae & 13 & 30 & 7 & \\
D. melanogaster & 289 & 9046 & 195 & 6.61 \\
H. sapiens & 486 & 23470 & 0 & 96.60 \\
\hline \hline
\end{tabular}

a scale-free architecture (Fig. 2, see also top half of Table I for other network descriptors).

Despite the complexity and large size of the networks, only the subgraphs containing recurrent connections are relevant for information processing according to the reservoir computing paradigm. ${ }^{22}$ To identify these substructures, we pruned the networks by eliminating all the strictly feedforward nodes. Spefically, the networks were simplified to a minimal recursive subgraph, i.e., a subgraph containing only the nodes and edges that form cyclic paths and the nodes that interconnect them. The pruning process consists in iteratively removing all the nodes with either no output or no input connections until no more nodes can be removed. This procedure leads to a directed recurrent graph of transcription factors, which will be referred to in what follows as the core or reservoir of the network (Fig. 3; see also bottom half of Table I for the topological properties of the reservoirs). Table II reports on the number of genes involved in each of the network substructures for the five organisms. As can be seen in the table, the recurrent cores in all five cases are much smaller, in terms of the number of genes involved, than the corresponding whole network.

In order to establish where each small subgraph of nodes is located within its network, we also show in Table II

a

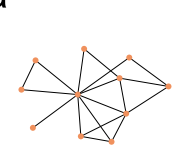

b
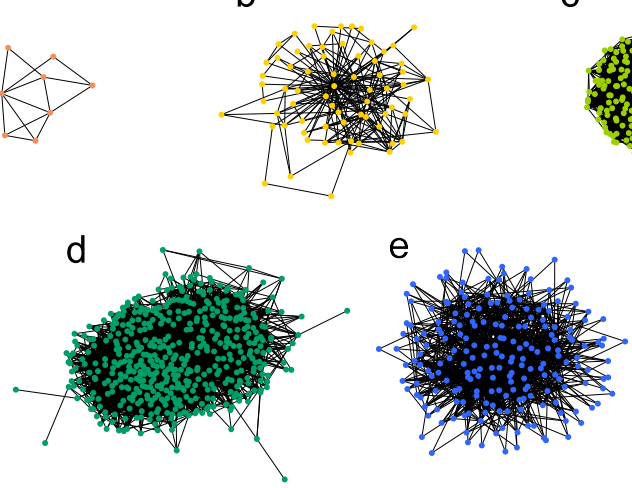

FIG. 3. Recurrent cores of the gene regulatory networks: Bacillus subtilis (a), Escherichia coli (b), Saccharomyces cerevisiae (c), Drosophila melanogaster (d), and Homo sapiens (e). All nodes in these subgraphs have both out-degree and in-degree larger than 0 .
TABLE II. Number of nodes in the different network substructures.

\begin{tabular}{lcccc}
\hline \hline & Total & Reservoir & Readout (terminal) & Other \\
\hline B. subtilis & 886 & 13 & $537(500)$ & 336 \\
E. coli & 3236 & 70 & $3133(3025)$ & 33 \\
S. cerevisiae & 6725 & 289 & $6436(6419)$ & 0 \\
D. melanogaster & 9432 & 486 & $8795(8721)$ & 151 \\
H. sapiens & 16354 & 207 & $13497(13449)$ & 2650 \\
\hline \hline
\end{tabular}

the fraction of genes located downstream of the reservoir (forming what we call the readout, following the organization depicted in Fig. 1). It can be observed that the vast majority of nodes are placed downstream of the recurrent core. The obvious implication of the location of the core is that most of the network is affected by its dynamics. It is worth noting again that by definition there are no recurrences outside the reservoir, and thus none of the readout nodes can affect back the reservoir. Furthermore, Table II also shows that a very large proportion of the readout nodes are terminal (i.e., nodes with no output connections), with the remaining fraction corresponding to transcription factors that are not part of the reservoir. The readout topology has thus limited complexity, which in turn limits its ability to process information, giving an even more central role to the recurrent core or reservoir, as we show below.

\section{NETWORK SIMULATION AND TRAINING}

The dynamics of the gene regulatory networks was simulated using a discrete time updating rule defined as

$$
x_{i, t+1}=\tanh \left(v_{i} z_{t}+\sum_{j=1}^{n} w_{i j} x_{j, t}\right),
$$

where we follow the notation shown in Fig. 4: $z_{t}$ is the system input at time $t, x_{i, t}$ is the state of node $i$ of the reservoir at time $t$ (corresponding to the elements of the reservoir state vector $X$ ), $n$ is the number of nodes in the reservoir, $w_{i j}$ is the weight of the link from the $j$ th to the $i$ th node (representing the elements of the weighted adjacency matrix $W$ ), and $v_{i}$ is the weight of the link from the input to the $i$ th node (corresponding to the elements of the input weight vector $V$ ). The values of $v_{i}$ are randomly chosen to be either -0.05 or 0.05 . At the same time, the values of $w_{i j}$ are real numbers drawn from a uniform distribution between -1 and 1 if the link exists, and 0 otherwise (if the sign of the interaction, i.e., activation vs repression, is known, the sign of $w_{i j}$ is set accordingly). Additionally, the $W$ matrix was normalized to have a spectral radius of 0.9 to ensure the echo-state property. ${ }^{13,16}$

Next, we asked if these recurrent cores are able to encode temporal information in their dynamics. To do so, we confronted them with the 10th order Nonlinear AutoRegressive Moving Average (NARMA) task, a memorydemanding benchmark commonly used in the reservoir computing context. ${ }^{23,24}$ The task consists in training a network to model the output of the 10th order NARMA system, ${ }^{25}$ a discrete time system where the input values $s(t)$ are drawn from the uniform distribution $\mathcal{U}(0,0.5)$, and the output $y(t)$ is 


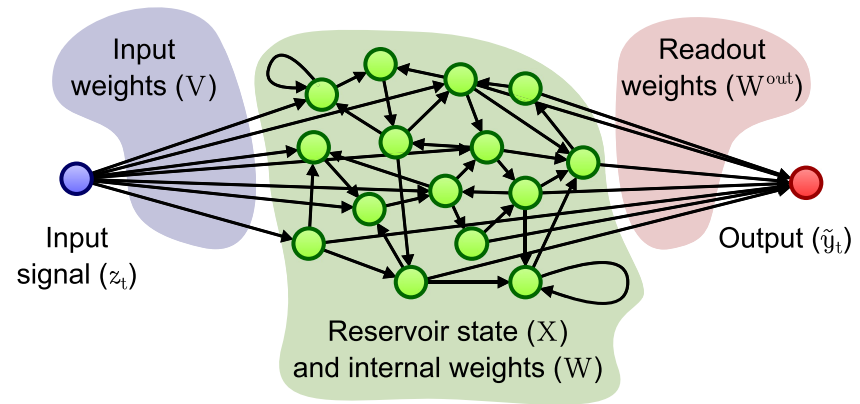

FIG. 4. Setup to test the memory of a network. A reservoir is built with a connectivity matrix $W$ extracted from the topology of the biological network. An input signal $z_{t}$ is applied to the nodes of the reservoir with different strengths, defined by the input weight vector $V$. Readout nodes compute a weighted sum of the state of the reservoir $X$. The weight vector $W^{\text {out }}$ is tuned so that the output $\tilde{y}_{t}$ of the readout approximates a target output signal $y_{t}$.

defined by

$$
\begin{aligned}
y(t+1)= & 0.3 y(t)+0.05 y(t) \sum_{i=0}^{9} y(t-i) \\
& +1.5 s(t-9) s(t)+0.1
\end{aligned}
$$

To test if the dynamics of the network cores can represent the temporally correlated NARMA input, the network was simulated with a time-varying random input $\left(z_{t}\right.$ in Fig. 4) feeding the $s(t)$ series in the system.

Then, a readout node was trained (i.e., the readout weights $W^{\text {out }}$ are adjusted) to reproduce the output $y_{t}$ of the 10th order NARMA system using only the instantaneous state of the network (Fig. 4). For each realization, a NARMA series of 10000 steps was generated, using 9000 of them for the training phase and 1000 to test the performance. Training is implemented by considering a readout node that computes a weighted sum of the state of the nodes from which it receives information (Fig. 4)

$$
\tilde{Y}=W^{\text {out }} X
$$

where $W^{\text {out }}$ is a vector of the $w_{i}^{\text {out }}$ weights given to the $i$ th node by the readout and $\tilde{Y}$ is a vector with all predicted outputs over time. To fit the $W^{\text {out }}$ weights we used ridge regression, a type of linear regression in which the regression coefficients are obtained from

$$
W^{\text {out }}=Y X^{T}\left(X X^{T}+\gamma^{2} I\right)^{-1},
$$

where $X^{T}$ is the transpose of $X, Y$ is a matrix with all expected outputs over time, $I$ is the identity matrix, and $\gamma$ is a regularization parameter. Ridge regression favors regression coefficients with smaller absolute values. In doing so, it introduces a certain bias, but on the other hand it also reduces the variance of the estimate. This allows estimating the parameters of a linear regression when the predictor variables are strongly correlated, making it a common readout choice in the context of reservoir computing. ${ }^{26}$

\section{NETWORK PERFORMANCE}

The challenge of the NARMA task is that the output of the 10th-order NARMA time series depends on the input and output values of the last 10 time steps. This information about

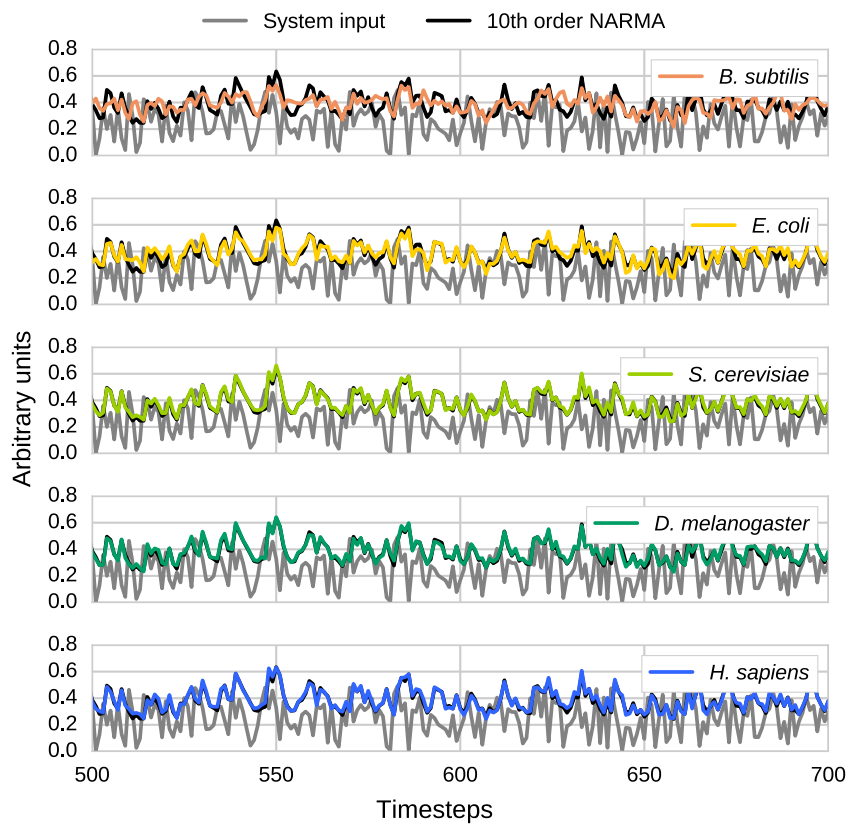

FIG. 5. Representative time series of the test phase of a 10th order NARMA task. For each biological network studied, a readout node was trained to reconstruct the output of the 10th-order NARMA system, using the state of the corresponding reservoir. Gray lines represent the random input of the system, black lines the actual output of the NARMA function, and colored lines the output reconstructed by the readout node.

the past must be encoded in the reservoir state for the output $\tilde{y}_{t}$ of the readout node to be able to accurately model the input. Figure 5 shows a representative time trace of the input signal $z_{t}$, the actual NARMA system output $y_{t}$, and the reconstruction $\tilde{y}_{t}$ obtained with each of the biological networks. The figure shows that the reconstructed output mimics closely the expected output for large enough reservoir sizes (bottom four rows, see Table II for core sizes).

We next compared the performance of our biological networks with the de facto standard topologies in the reservoir computing literature:

- Echo State Network with fixed mean degree (kESN): Erdös-Rényi random network with the same mean degree $\left(2 \times n_{\text {edges }} / n_{\text {nodes }}\right)$ as the topology of the corresponding biological network.

- Echo State Network with fixed network density (dESN): Erdös-Rényi random network with the same network density-i.e., fraction of existing links over all possible ones- $\left(n_{\text {edges }} / n_{\text {nodes }}^{2}\right)$ as the topology of the corresponding biological network.

- Simple Cycle Reservoir (SCR): a directed circular graph, which is the simplest topology that can work as a computational reservoir. ${ }^{22}$

Note that for control networks with the same number of nodes as the problem topology, kESN is equal to dESN. This is not the case, however, when the number of nodes changes.

To quantify the network performance, we used the Normalized Root Mean Squared Error (NRMSE) between the reconstructed and the expected output signals, which is 


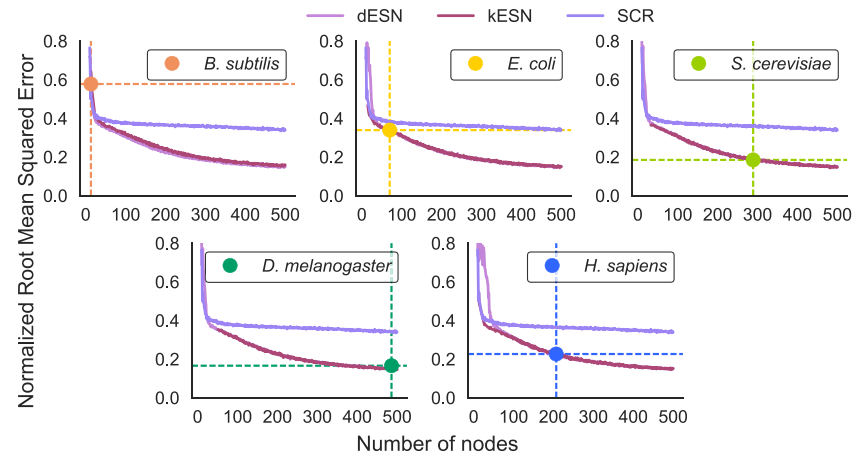

FIG. 6. Performance of the biological reservoirs compared with control topologies. Performance is evaluated with the Normalized Root Mean Squared Error (NRSME) between expected and reconstructed outputs. The NRMSE value shown for each biological network topology corresponds to the median of 10000 trials (with edge weights and data series randomization). The values plotted for each control network (dESN, kESN, and SCR) correspond to the median value of 100 trials for each network size from 10 to 500 nodes.

defined as

$$
\mathrm{NRMSE}=\sqrt{\frac{\left\langle[\tilde{y}(t)-y(t)]^{2}\right\rangle_{t}}{\left\langle\left[y(t)-\langle y(t)\rangle_{t}\right]^{2}\right\rangle_{t}}},
$$

where $\tilde{y}(t)$ is the output predicted by readout, $y(t)$ is the output of the actual NARMA system, and $\langle\cdot\rangle_{t}$ indicates the mean over time.

Figure 6 shows the median NRMSEs achieved by our reservoirs and compares them with control topologies of diverse core sizes. The control networks take the form of random (Erdös-Renyi) echo-state networks for which the network density (dESN) or mean degree (kESN) is kept constant (and equal to their biological counterparts) as the network size varies. We also include results for simple cycle reservoirs (SCRs), linear cyclic reservoirs with the minimum recurrence that allows them to operate as echo-state networks. ${ }^{22}$ As the figure shows, all the biological cores perform as well as the random dESN and kESN control networks of the same size, and for large enough core sizes ( $S$. cerevisiae, D. melanogaster, $H$. sapiens), the performance is much better than the corresponding SCR. In fact, differences between SCR and both the biological networks and ESN variants increase with size within the interval analyzed. Our results thus show that even though biological networks are not random (since certain genes are necessarily more central than others, due to their biological function within cells), they can process information at near optimal levels. In other words, gene regulatory networks combine functional specificity and global computational performance.

Our results also suggest that the different performance of each GRN is related with their size. In this regard, it is worth noting that despite the fact that the number of edges in the control networks scales linearly with size for kESNs and quadratically for dESNs, they show similar performance to each other for all the range of sizes. That discards any major effect of the number of edges in the performance of the reservoir in these conditions.

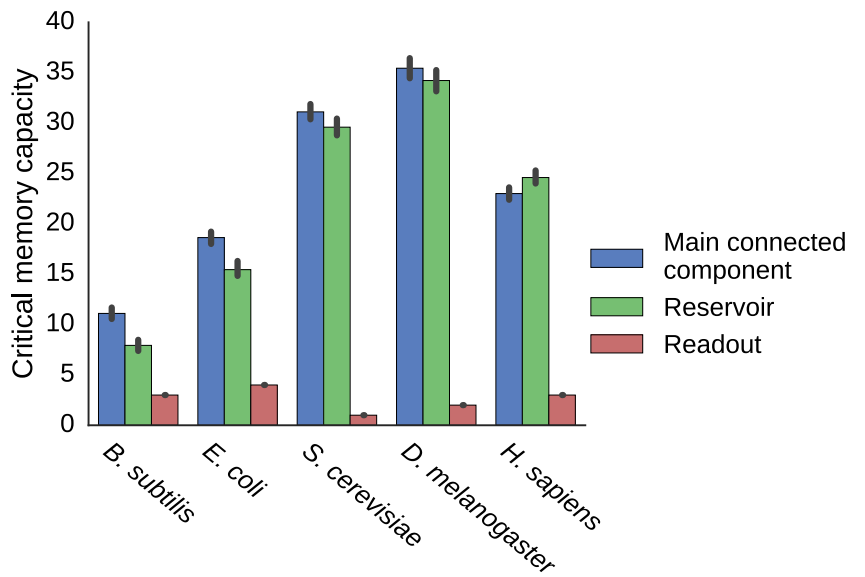

FIG. 7. Critical memory capacity of the different network substructures. Results are shown for the reservoir (green) and readout (red) substructures and for the largest connected component-which comprises the other two-(blue), for each of the gene regulatory networks analyzed. Median values are shown $(n=30$ trials). The error bars indicate the $98 \%$ confidence interval (CI) computed by bootstrapping.

\section{MEMORY CAPACITY}

To quantify the amount of temporal information that our networks can store, we computed their critical memory capacity (maximum number of past time steps that can be recovered with a given accuracy) as a variation of the concept of short-term memory capacity. ${ }^{27,28}$ Specifically, we simulated the network with a single input node feeding a signal $u(t)$ drawn from a random uniform distribution between -1 and 1 . Then, a ridge-regression node was trained to obtain an output $\tilde{y}(t)$ that aims to reconstruct a delayed version of the input signal $u(t-k)$. The $k$-delay memory capacity $\left(M C_{k}\right)$ is then defined as

$$
M C_{k}=\frac{\operatorname{cov}^{2}[u(t-k), \tilde{y}(t)]}{\sigma^{2}[u(t-k)] \cdot \sigma^{2}[\tilde{y}(t)] .}
$$

The short-term memory capacity is typically defined as $M C=$ $\sum_{k=1}^{\infty} M C_{k}$, where the infinite sum is approximated by a finite sum of sufficient length. ${ }^{27,28}$ The limitation in that case is that the time series needs to be orders of magnitude longer than the size of the network to ensure that $\lim _{k \rightarrow \infty} M C_{k}=0$, otherwise $M C_{k}$ will never reach 0 and $M C$ will never converge. Since we were dealing with fairly large networks, computing the short-term memory capacity with a reasonable precision was not feasible. As an alternative measure, we defined the critical memory capacity $k^{*}$ as the maximum delay $k$ that fulfills $M C_{k}>0.5$.

For each gene regulatory network, we measured the critical memory capacity of three subnetworks: the recurrent reservoir, the readout, and the largest connected component (which contains the first two). The results, shown in Fig. 7, confirm that most of the ability of our networks to encode history is provided by their reservoirs (the green and blue bars in the figure have very similar heights in all cases). In contrast, the readouts have much lower memory capacities, in spite of being much larger in size than the reservoirs (see again Table II). The critical memory capacities of the readouts are mainly determined by the length of the longest path (Fig. 8), as expected from their feedforward structure. These results 
confirm that the recurrent cores are responsible for most of the capacity to dynamically store temporal information of the gene regulatory networks.

\section{BIOLOGICAL INPUTS}

We have considered so far that information is introduced in the reservoirs via randomly selected nodes. However, realistic biological inputs act upon specific sets of nodes. To determine whether the reservoir computing paradigm holds in the presence of such realistic inputs, we identified nodes from the reservoir that belonged to particular stress-response pathways and studied the effect of the corresponding stresses. We worked specifically with the E. coli reservoir in order to keep a balance between methodological tractability and network performance. Specifically, the size of the E. coli reservoir is small enough to enable systematic computational analysis (but not too small, which is the case of the B. subtilis network). This fact, together with the good annotation of the regulatory networks of this organism, allowed us to manually research each node.

We analyzed which of the 70 genes present in the $E$. coli reservoir were known to be affected by signaling pathways that react to different stress classes. Biological stresses of seven different classes were considered, namely, presence of antibiotics, anaerobiosis, osmotic stress, oxidative stress, starvation, changes in temperature, and changes in $\mathrm{pH}$. Using the annotations of the Ecocyc database, ${ }^{18}$ we manually set a confidence score to each possible stress-gene interaction. This score indicates the level of evidence supporting that the product of a given gene is affected by a signaling pathway in response to a given stress. Both post-transcriptional and transcriptional regulations were considered to determine if a signaling pathway could reach a given gene, as long as they were not already included in the network structure (i.e., only transcriptional interactions coming from outside the reservoir a

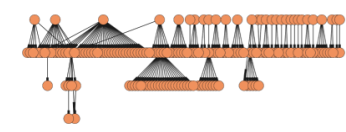

b

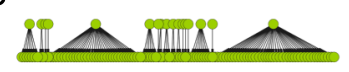

C

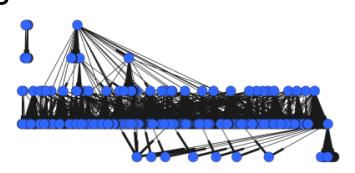

d

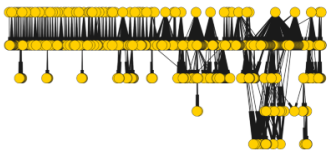

e

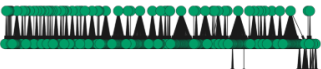

$f$

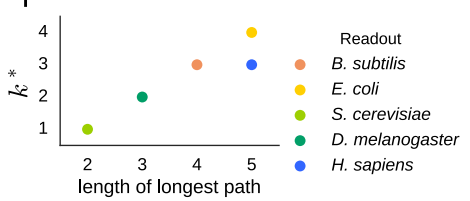

FIG. 8. Topology and critical memory capacity of the readout structures of the gene regulatory networks. Hierarchical representation of the readout of the five gene regulatory networks: B. subtilis (a), E. coli (b), S. cerevisiae (c), $D$. melanogaster (d), and $H$. sapiens (e). Nodes are ordered in layers from top to bottom according to the length of the longest path reaching them from the reservoir. Panel $(\mathrm{F})$ shows the relation between the length of the longest path in each of the readouts and their critical memory capacity $k^{*}$, which measures the number of time steps in the past that can be remembered with a certain precision in the system dynamics.
TABLE III. Number of nodes affected by stress type for each evidence threshold.

\begin{tabular}{lrrrrr}
\hline \hline & \multicolumn{5}{c}{ Evidence threshold } \\
\cline { 2 - 6 } Stress type & 1 & 2 & 3 & 4 & 5 \\
\hline Antibiotics & 16 & 10 & 6 & 3 & 1 \\
Anaerobiosis & 6 & 6 & 5 & 3 & 2 \\
Osmotic stress & 13 & 13 & 7 & 6 & 3 \\
Oxidative stress & 17 & 17 & 13 & 9 & 5 \\
Starvation & 30 & 29 & 25 & 18 & 10 \\
Changes in temperature & 8 & 8 & 7 & 6 & 5 \\
Changes in pH & 15 & 14 & 10 & 7 & 1 \\
Any stress & 58 & 57 & 52 & 43 & 29 \\
\hline \hline
\end{tabular}

were considered). Table III lists the number of nodes that are considered to receive information from every stress with a confidence score equal or larger than a given threshold.

Using the classification described above, an input weight vector $V$ was constructed for each stress class and confidence threshold. Given a threshold, all interactions with lower score were set to zero while the others were initiated normally. Additionally, the sign of each entry was set to be positive (negative) if the interaction was known to produce an activation (repression) of the gene, and randomly set otherwise.

We then subjected the E. coli reservoir to a variation of the NARMA test, in which the stress signals act solely upon the input genes selected above. Figure 9 shows the resulting NRMSE as a function of the number of input nodes for the different stresses (encoded with distinct colors) and for all confidence thresholds (which correspond to different sizes, according to Table III). As a control, the figure also shows the NRSME obtained by applying the input signal to random sets of nodes (gray line). The most obvious conclusion from these results is that biologically realistic inputs are as efficient as randomly selected nodes at encoding information in the reservoir. Also, the precision of the system increases monotonically with the size of the input set and eventually saturates. Besides, it is noteworthy that although the different stress signaling pathways affect different sets of nodes, their ability to introduce information in the system is comparable. This highlights the fact that memory is encoded in the network in a delocalized manner, without depending on specialized circuits or structures.

\section{EVOLUTIONARY TRAINING}

In machine learning applications, reservoir computing usually relies on ridge regression, described above, to train the readout weights. Cellular networks, however, should use biologically realistic means to perform this training. A reasonable possibility is that the readout weights are tuned through evolutionary processes. To assess this possibility, we used here an evolutionary algorithm. Starting from a first generation of random candidate weight vectors, we iteratively generate new generations by duplicating and introducing variations to the best performing solutions in the current generation.

Specifically, we used the implementation of the Covariance Matrix Adaptation Evolutionary Strategy (CMA-ES) 


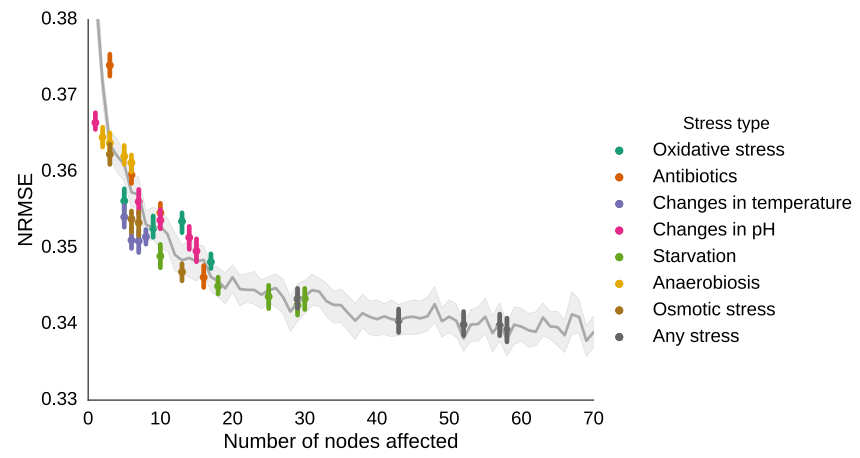

FIG. 9. Efficiency of signaling pathways feeding an input signal to the reservoir. Median NRMSE obtained in the NARMA test using as input nodes those genes affected by signaling pathways that react to different stress types. The gray line marks the NRMSE obtained when applying the input to a random set of nodes of a given size. The median values are shown of 1000 replicates for each random input size and 2000 for each biological input set. Error bars and shaded area indicate the $98 \%$ CI computed by bootstrapping.

algorithm ${ }^{29}$ provided in the package "Distributed Evolutionary Algorithms in Python" (DEAP). ${ }^{30}$ The CMA-ES algorithm learns the covariance matrix of mutations in successful individuals so that beneficial mutations are sampled more often. This approach reduces the computational cost while preserving the biological relevance.

Using the E. coli reservoir, we evolved a population of single-node readouts with a selective pressure to predict the 10th order NARMA system. To that end, we applied to the $E$. coli reservoir a single input $s(t)$ drawn from a random uniform distribution $\mathcal{U}(0,0.5)$. The input vector $V$ was defined so that the signal would only reach genes known to be affected by at least one stress type with a confidence score of 3 or more. A population of readout nodes, represented by their weight vectors $W^{\text {out }}$, was then let to compete and evolve, giving a selective advantage to the ones that reproduced better the output of the NARMA system. For each generation in the evolutionary process a new realization of the $s(t)$ input signal was used, recomputing the reservoir dynamics and the expected output. The internal weights of the reservoir $W$ and the input weights $V$ were kept constant for the whole simulation. Figure 10 features a representative instance of such evolutionary processes (green), compared with the behavior of a standard ridge regression training method (purple). The performance of both types of readouts was practically indistinguishable after 2000 generations, indicating that evolution can, indeed, tune a readout structure to read the temporal information stored in a reservoir.

\section{APPROACHES TO EXPERIMENTAL VALIDATION}

The results described so far suggest that the recurrent architecture of gene regulatory networks enables cells to process complex dynamic information. Experimentally validating this hypothesis requires means to quantify the ability of cells to remember changes in their environment. This can be accomplished by subjecting cells to different environmental histories and quantifying the mutual information between that history and the behavior of the cells (as measured by their growth rates and gene expression levels). This approach has

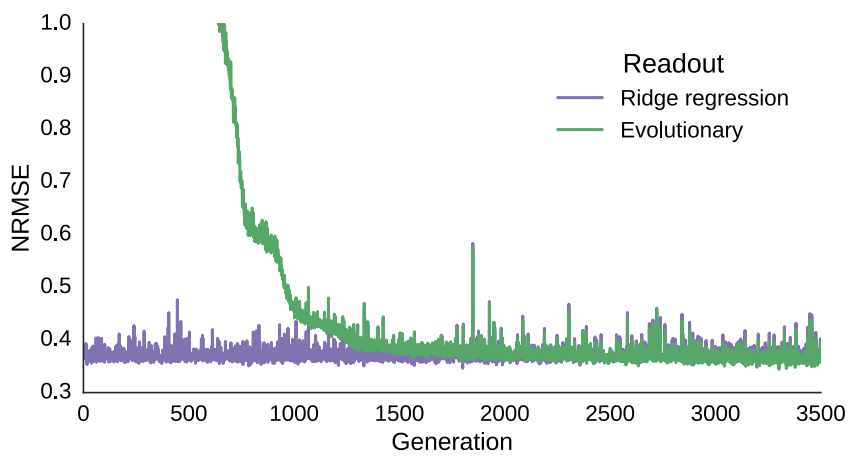

FIG. 10. Training of a readout through an evolutionary process. NRMSE during the evolutionary training process of a readout for the $E$. coli reservoir. The weights of the reservoir node were trained using the CMA-ES ${ }^{29}$ algorithm to model the 10th order NARMA system Conceptually, a population of 200 candidate solutions is let to evolve while giving a selective advantage to those obtaining a lower NRMSE. The green line shows the NRMSE of the centroid of the best solutions in each generation. The purple line shows the NRMSE obtained in the same situation by a readout trained using standard ridge regression.

already been implemented experimentally with binary environmental changes ${ }^{9}$ and could be extended to more complex input dynamics in a relatively straightforward way. With this possibility in mind, we now examine how the critical memory capacity, introduced in Sec. $\mathrm{V}$, depends in a unique manner on the particular properties of the E. coli reservoir studied so far.

We first consider the effect of a global scaling of the connectivity matrix of the reservoir. This would be reflected in a change in its spectral radius, which is known to play a crucial role in the computational capabilities of echo-state networks. ${ }^{16}$ Such perturbation could be accomplished experimentally by altering in a global manner the gene expression potential of cells (varying in a controlled manner the levels of resource cellular components such as RNA polymerases, housekeeping sigma factors, or ribosomes). Figure 11(a) shows that the critical memory capacity of the E. coli reservoir is maximal for a spectral radius around 1 , in contrast with a purely feedforward network with the same number of nodes and links, for which the memory capacity is constant, depending only on the distance between the input and output nodes, as shown in Fig. 8 for the readout layer. Thus if the cell processes information according to the reservoir computing paradigm, its memory capacity should vary non-monotonically with its global expression capacity. An interest open question in this case will then be if natural networks are tuned to operate near the maximum memory capacity.

It is difficult to vary common gene-expression resources in vivo in a controlled way while avoid pleiotropic effects. We thus turned to studying the effects of local perturbations, in particular mutations that eliminate specific transcriptional interactions in the network. The inhomogeneous nature of the connectivity of the E. coli reservoir [Fig. 2(b)] implies that not all links have the same importance in determining the amount of memory of the network. This is shown in Fig. 11(b), in which the links have been eliminated one by one, with the corresponding critical memory capacity being plotted in the $y$ axis. The links are ranked left to right from stronger to weaker effect (in terms of reducing the critical memory capacity). The 
a

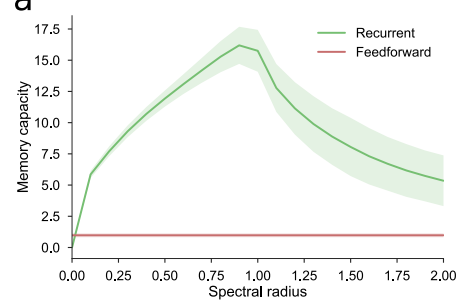

C

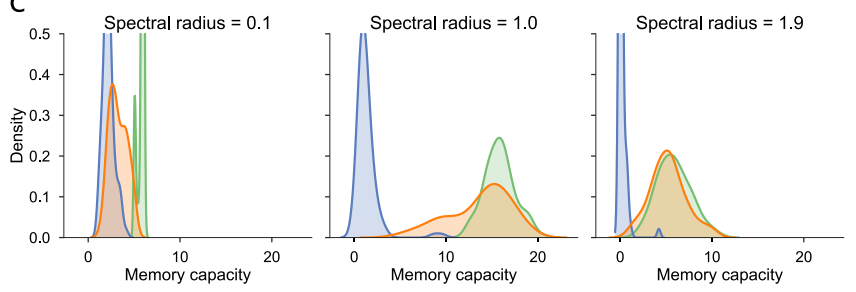

b

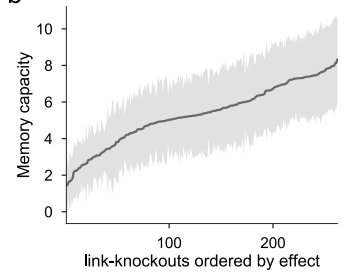

Spectral radius $=1.9$
FIG. 11. Effect of reservoir perturbations on memory capacity. (a) Critical memory capacity as a function of the spectral radius of the E. coli recurrent reservoir (green) and corresponding measurement for a feedforward network with the same number of nodes and links (red). The green shadowing represents the standard deviation of 500 realizations of the network with random intra-reservoir weights. (b) Critical memory capacity resulting from knocking out one by one all interactions in the E. coli reservoir, ranked according to their effect, for spectral radius 0.7 . The gray shadowing represents the standard deviation of 100 realizations of the network. (c) Distribution of critical memory capacities (across 100 realizations and approximated via a kernel density estimation) for three values of the spectral radius. The full reservoir case is shown in green, and those of the networks resulting from knocking out the links with strongest and weakest effects (first and last data points in panel b) are shown in blue and orange, respectively.

difference between the effects of the most and least important links is maximized again when the spectral radius is closer to 1 [Fig. 11(c), with the most important link being shown in blue and the least important one in orange], but the decrease of the memory capacity with respect to the wild-type situation [green in Fig. 11(c)] should be observable experimentally irrespective of the specific value of the spectral radius.

\section{DISCUSSION}

Our results suggest that gene regulatory networks can store and process temporal information through a division of labor that allows cells to separate the processes of memory encoding and decision making in two distinct regions of the network. The first region, the reservoir, has recurrences-i.e., cyclic paths-that give it a fading memory property so that it can efficiently encode recent history. The latter region, the readout, has a feedforward or acyclic structure and uses the information it receives from the reservoir to make a classification or prediction. This separation of roles allows the system to process temporal information while still being very efficient when learning new tasks. ${ }^{15}$

To support this hypothesis, we analyze the gene regulatory networks of five evolutionary distant organisms, focusing on Escherichia coli for biological insight. First, the topology of all five networks matches the structural characteristics of a reservoir computing system. Second, we show that these loosely defined reservoir structures are able to encode in their dynamics an amount of temporal information that is nontrivial given their sizes. As a matter of fact, for all networks the reservoir is one to two orders of magnitude smaller than the readout, and yet its critical memory capacity is up to around 30 times higher. Moreover, in the case of E. coli, biological signals relevant for the cell (specifically, physiological stresses) arriving at the reservoirs at different locations are encoded with similar performance, indicating that the ability to store temporal information is distributed in the reservoir. This independence of the specific entry point of the information in the system confers robustness to failure: while some of the input streams may get compromised, it is unlikely that a large group of them would fail simultaneously. We also show that evolution can produce readout structures that are able to decode the state of a reservoir, as long as temporal information provides a selective advantage. Finally, we discuss approaches to experimentally validate the mechanism put forward in this paper.

Non-recurrent gene network architectures have been proposed in the past as mechanisms of information integration and storage, ${ }^{31,32}$ associative learning, ${ }^{33,34}$ and cellular decision making. ${ }^{35,36}$ However, processing of time-dependent information requires recurrent topologies such as the ones investigated in this paper. The nonconventional computation framework proposed here also implies that the integration of information is distributed across the network in large and diffuse structures with well-defined functional roles. A similar connection between gene regulatory networks and reservoir computing systems was hinted by Jones et al. ${ }^{37}$ However, that study uses as putative reservoir a gene regulatory network that does not include any recurrences other than self-regulations of some scarcely interconnected nodes, and the system is tested with a task that does not require memory.

Even though it is clear that cells benefit from anticipating the environment, we know no example yet of a single cellular system that processes complex temporal information in nature. The most studied types of anticipation involving periodic ${ }^{2,3}$ and sequential ${ }^{4,5}$ events are mechanistically fairly simple. However, ad hoc experiments have shown that Physarum polycephalum, also known as slime mold, and Plasmodium cudatum can learn very efficiently new temporal structures. Slime mold, in particular, can anticipate a shock after experiencing a single series of three ten-minute low-temperature shocks at one-hour intervals. Moreover, if the organism experiences a new shock several hours later, it pre-emptively reacts to the two missing following shocks. ${ }^{38}$ Similarly, $P$. cudatum can learn that an electric shock follows an innocuous vibratory or luminous stimulus. ${ }^{39,40}$ All these results hint at capabilities to learn temporal structures larger than what can be easily explained with current models.

We propose that cells can process temporal information and anticipate their environment by using their regulatory networks as computational reservoirs. To that end, here we explored the potential of transcriptional networks to encode the recent history of cells, but other regulatory networks such as protein-protein interaction or metabolic networks may play a similar role. The combination of the different time scales (minutes or seconds) and learning mechanisms (evolution, chromatin regulation for transcriptional reservoirs, or expression regulation for post-transcriptional reservoirs) could give rise to much richer behaviors. 
In our study, the nonlinear dynamics of the networks have been largely simplified with a formalism used for neural networks. The real dynamics, with more realistic interactions and different time scales for each gene, would add more complexity to the network behavior and increase the memory of the system. ${ }^{41-43}$ Furthermore, the interaction of layers of regulatory networks with different time scales-e.g., transcriptional, protein protein interaction or metabolic networks-also could increase the memory capacity of the system. ${ }^{42,44}$ Far from invalidating our results, our simplification of the dynamics makes our tests more stringent. Additionally, the NARMA task is known to be highly demanding, as it requires a significant level of precision in the results. Probably life does not need to be as precise.

\section{ACKNOWLEDGMENTS}

We thank Rosa Martinez-Corral for useful comments. This work was supported by the Spanish Ministry of Economy and Competitiveness and FEDER (Project No. FIS201566503-C3-1-P), and by the Generalitat de Catalunya (Project No. 2017 SGR 1054). J.G.O. also acknowledges support from the ICREA Academia programme and from the "María de Maeztu" Programme for Units of Excellence in R\&D (Spanish Ministry of Economy and Competitiveness, MDM-20140370).

${ }^{1}$ S. Postnova, K. Voigt, and H. A. Braun, "A mathematical model of homeostatic regulation of sleep-wake cycles by hypocretin/orexin," J. Biol. Rhythms 24, 523-535 (2009).

${ }^{2}$ S. S. Golden, M. Ishiura, C. H. Johnson, and T. Kondo, "Cyanobacterial circadian rhythms," Annu. Rev. Plant Physiol. Plant Mol. Biol. 48, 327-354 (1997).

${ }^{3}$ T. Mori and C. H. Johnson, "Circadian programming in cyanobacteria," Semin. Cell Dev. Biol. 12, 271-278 (2001).

${ }^{4}$ I. Tagkopoulos, Y.-C. Liu, and S. Tavazoie, "Predictive behavior within microbial genetic networks," Science 320, 1313-1317 (2008).

${ }^{5}$ A. Mitchell, G. H. Romano, B. Groisman, A. Yona, E. Dekel, M. Kupiec, O. Dahan, and Y. Pilpel, "Adaptive prediction of environmental changes by microorganisms," Nature 460, 220-224 (2009).

${ }^{6}$ A. Rodaki, I. M. Bohovych, B. Enjalbert, T. Young, F. C. Odds, N. A. Gow, and A. J. Brown, "Glucose promotes stress resistance in the fungal pathogen candida albicans," Mol. Biol. Cell 20, 4845-4855 (2009).

${ }^{7}$ S. Schild, R. Tamayo, E. J. Nelson, F. Qadri, S. B. Calderwood, and A. Camilli, "Genes induced late in infection increase fitness of Vibrio cholerae after release into the environment," Cell Host Microbe 2, 264-277 (2007); arXiv:NIHMS150003.

${ }^{8}$ R. Dhar, R. Sägesser, C. Weikert, and A. Wagner, "Yeast adapts to a changing stressful environment by evolving cross-protection and anticipatory gene regulation," Mol. Biol. Evol. 30, 573-588 (2013).

${ }^{9}$ D. M. Wolf, L. Fontaine-Bodin, I. Bischofs, G. Price, J. Keasling, and A. P. Arkin, "Memory in microbes: Quantifying history-dependent behavior in a bacterium," PloS One 3, e1700 (2008).

${ }^{10}$ T. I. Lee, N. J. Rinaldi, R. François, D. T. Odom, Z. Bar-Joseph, G. K. Gerber, N. M. Hannett, C. T. Harbison, C. M. Thompson, I. Simon, J. Zeitlinger, E. G. Jennings, H. L. Murray, D. B. Gordon, B. Ren, J. J. Wyrick, J.-B. Tagne, T. L. Volkert, E. Fraenkel, D. K. Gifford, and R. A. Young, "Transcriptional regulatory networks in Saccharomyces cerevisiae," Science 298, 799-804 (2009).

${ }^{11}$ A. Martínez-Antonio and J. Collado-Vides, "Identifying global regulators in transcriptional regulatory networks in bacteria," Curr. Opin. Microbiol. 6, 482-489 (2003).

${ }^{12}$ D. Verstraeten, B. Schrauwen, M. D'Haene, and D. Stroobandt, “An experimental unification of reservoir computing methods," Echo State Networks and Liquid State Machines, Neural Netw. 20, 391-403 (2007).

${ }^{13} \mathrm{H}$. Jaeger, "The 'echo state' approach to analysing and training recurrent neural networks," Tech. Rep. (Fraunhofer Institute for Autonomous Intelligent Systems, 2001).
${ }^{14}$ W. Maass, T. Natschlager, and H. Markram, "Real-time computing without stable states: A new framework for neural computation based on perturbations," Neural Comput. 14, 2531-2560 (2002).

${ }^{15}$ D. V. Buonomano and W. Maass, "State-dependent computations: Spatiotemporal processing in cortical networks," Nat. Rev. Neurosci. 10, 113-125 (2009).

${ }^{16}$ M. Lukoševičius and H. Jaeger, "Reservoir computing approaches to recurrent neural network training," Comput. Sci. Rev. 3, 127-149 (2009).

${ }^{17}$ N. Sierro, Y. Makita, M. de Hoon, and K. Nakai, "DBTBS: A database of transcriptional regulation in Bacillus subtilis containing upstream intergenic conservation information," Nucleic Acids Res. 36, D93-D96 (2008).

${ }^{18}$ I. M. Keseler et al., "EcoCyc: A comprehensive database of Escherichia coli biology," Nucleic Acids Res. 39, D583-D590 (2011).

${ }^{19}$ M. C. Teixeira et al., "The YEASTRACT database: An upgraded information system for the analysis of gene and genomic transcription regulation in Saccharomyces cerevisiae," Nucleic Acids Res. 42, D161-D166 (2014).

${ }^{20} \mathrm{~S}$. Roy et al., "Identification of functional elements and regulatory circuits by Drosophila modENCODE," Science (New York, N.Y.) 330, 1787-1797 (2010).

${ }^{21}$ M. B. Gerstein et al., "Architecture of the human regulatory network derived from ENCODE data," Nature 489, 91-100 (2012).

${ }^{22}$ A. Rodan and P. Tino, "Minimum complexity echo state network," IEEE Trans. Neural Netw. 22, 131-144 (2011).

${ }^{23}$ H. Jaeger, "Adaptive nonlinear system identification with echo state networks,” Adv. Neural Inf. Process. Syst. 15, 609-616 (2002).

${ }^{24}$ L. Appeltant, M. Soriano, G. Van der Sande, J. Danckaert, S. Massar, J. Dambre, B. Schrauwen, C. Mirasso, and I. Fischer, "Information processing using a single dynamical node as complex system," Nat. Commun. 2, 468 (2011); arXiv:1111.7219.

${ }^{25}$ A. F. Atiya and A. G. Parlos, "New results on recurrent network training: Unifying the algorithms and accelerating convergence," IEEE Trans. Neural Netw. 11, 697-709 (2000).

${ }^{26}$ F. Wyffels, B. Schrauwen, and D. Stroobandt, "Stable output feedback in reservoir computing using ridge regression," in Artificial Neural Networks - ICANN 2008, Lecture Notes in Computer Science, Vol. 5163, edited by V. Kůrková, R. Neruda, and J. Koutník (Springer, Berlin, 2008), pp. 808-817.

${ }^{27}$ H. Jaeger, "Short term memory in echo state networks," Tech. Rep. (German National Research Center for Information Technology, 2001).

${ }^{28}$ J. Boedecker, O. Obst, J. T. Lizier, N. M. Mayer, and M. Asada, "Information processing in echo state networks at the edge of chaos," Theory Biosci. 131, 205-213 (2012).

${ }^{29}$ F. Jiang, H. Berry, and M. Schoenauer, "Supervised and evolutionary learning of echo state networks," in Parallel Problem Solving from Nature, edited by G. Rudolph, T. Jansen, N. Beume, S. Lucas, and C. Poloni (Springer, Berlin, 2008), pp. 215-224.

${ }^{30}$ C. Gagn, "DEAP: Evolutionary algorithms made easy," J. Mach. Learn. Res. 13, 2171-2175 (2012).

${ }^{31}$ D. Bray, "Protein molecules as computational elements in living cells," Nature 376, 307 (1995).

${ }^{32}$ B. Scheres and W. H. Van Der Putten, "The plant perceptron connects environment to development," Nature 543, 337-345 (2017).

${ }^{33}$ S. McGregor, V. Vasas, P. Husbands, and C. Fernando, "Evolution of associative learning in chemical networks," PLoS Comput. Biol. 8, e1002739 (2012).

${ }^{34}$ M. Sorek, N. Q. Balaban, and Y. Loewenstein, "Stochasticity, bistability and the wisdom of crowds: A model for associative learning in genetic regulatory networks," PLoS Comput. Biol. 9, e1003179 (2013).

${ }^{35}$ R. Bates, O. Blyuss, A. Alsaedi, and A. Zaikin, "Effect of noise in intelligent cellular decision making," PloS One 10, e0125079 (2015).

${ }^{36}$ S. Filicheva, A. Zaikin, and O. Kanakov, "Dynamical decision making in a genetic perceptron," Phys. D: Nonlinear Phenom. 318, 112-115 (2016).

${ }^{37}$ B. Jones, D. Stekel, J. Rowe, and C. Fernando, "Is there a liquid state machine in the bacterium escherichia coli?," in 2007 IEEE Symposium on Artificial Life (IEEE, 2007), pp. 187-191.

${ }^{38}$ T. Saigusa, A. Tero, T. Nakagaki, and Y. Kuramoto, "Amoebae anticipate periodic events," Phys. Rev. Lett. 100, 1-4 (2008).

${ }^{39}$ T. M. Hennessey, W. B. Rucker, and C. G. McDiarmid, "Classical conditioning in paramecia," Animal Learn. Behav. 7, 417-423 (1979). 
${ }^{40}$ H. L. Armus, A. R. Montgomery, and R. L. Gurney, "Discrimination learning and extinction in Paramecia (P. caudatum)," Psychol. Rep. 98, 705-711 (2006).

${ }^{41}$ L. Büsing, B. Schrauwen, and R. Legenstein, "Connectivity, dynamics, and memory in reservoir computing with binary and analog neurons," Neural Comput. 22, 1272-1311 (2010).

${ }^{42}$ J. Dambre, D. Verstraeten, B. Schrauwen, and S. Massar, "Information processing capacity of dynamical systems," Sci. Rep. 2, 514 (2012).
${ }^{43}$ G. Tanaka, R. Nakane, T. Yamane, D. Nakano, S. Takeda, S. Nakagawa, and A. Hirose, "Exploiting heterogeneous units for reservoir computing with simple architecture," in International Conference on Neural Information Processing (Springer, 2016), Vol. 1, pp. 187-194.

${ }^{44} \mathrm{C}$. Gallicchio and A. Micheli, "Deep reservoir computing: A critical analysis," in European symposium on artificial neural networks, computational intelligence and machine learning, April (i6doc.com, 2016), pp. 27-29. 